\title{
Face Expression Recognition using CNN
}

\author{
Ashna Namira, Aravind Naik, Nikhil Floyd Dsouza
}

Computer Science Engineering, Srinivas Institute of Technology, Mangalore, Karnataka, India

\section{Article Info}

Volume 7, Issue 4

Page Number: 235-240

\section{Publication Issue :}

July-August-2021

\section{Article History}

Accepted : 10 July 2021

Published : 18 July 2021

\begin{abstract}
The emotions evolved in face have an excellent influence on decisions and arguments about various subjects. In psychological theory, emotional states of an individual are often classified into six main categories: surprise, fear, disgust, anger, happiness and sadness. Automatic extraction of those emotions from the face images can help in human computer interaction also as many other applications. Machine learning algorithms and particularly deep neural network can learn complex features and classify the extracted patterns. In this paper, a deep learningbased framework is used for human emotion recognition. The proposed framework uses the feature extraction then a Convolutional Neural Network (CNN) for classification. The experimental results show that the proposed methodology increases both of the speed training process of CNN and therefore the recognition accuracy.
\end{abstract}

Keywords: Inductive Learning Algorithm (ILA), XCeption Algorithm

\section{INTRODUCTION}

Face recognition as a identity verification technology is one in every of the recent topics among the sector of pattern recognition,image process, machine vision, neural network and science in recent years. it's a crucial application of Image processing thanks to its use in several fields. Face recognition cites to a static face image or dynamic video, which may verify the identity of one or multiple individuals employing a face information with many famous identities.

As a crucial facet of biometric authentication, there are wide application prospects within the fields of identity authentication, security observance and human pc interaction.Deep learning ways are able to leverage massive datasets of faces and learn made and compact representations of faces, permitting trendy models to first perform as-well so to outstrip the face recognition potentialities of human beings. compared to ancient machine learning approaches, deep learning-based methods have shown a great deal higher performances in terms of accuracy and speed of process in image recognition. CNNs have evidenced terribly effective in areas like image recognition and classification. CNNs are a sort of feed-forward neural networks created of several layers. This deep learning methodology can extract very abstract options by itself by finding the patterns within the image. 


\section{LITERATURE SURVEY}

Yongmian Zhang et al. [1] explored the usage of the multisensory data fusion method with Dynamic Bayesian networks (DBNs) for modelling and knowhow of the temporal behaviour of facial expressions withinside the face sequences formed .

Irene Kotsia and Ioannis Pitas [2] defined novel strategies for countenance popularity the usage of SVMs for countenance popularity. The consumer initializes style of the Candide grids nodes at the facial picture depicted on the number one body of the picture sequence.

Yongmian Zhang et al. [3] proposed probabilistic framework for fact duplicate of dynamic facial expressions on a face version with MPEG-4 facial animation parameters (FAPs) wherein accomplishing extraordinarily low bitrate in statistics transmission. Casted the FAPs and facial motion writing right into a dynamic Bayesian network (DBN) to account for uncertainties in FAP extraction and to version the dynamic evolution correct expressions. Thereby ensuing in manufacturing of practical expressions.

Yongqiang $\mathrm{Li}$ et al. [4] said facial characteristic factors round every facial component, i.e., eyebrow, mouth, etc., to seize the complete exact face form data. Facial motion gadgets which can be described withinside the facial motion writing represents the contraction of a particular set of facial muscles.

Muhammad Hameed Siddiqi et al. [5] proposed Stepwise Linear Discriminant Analysis (SWLDA). SWLDA specializes in deciding on the localized functions from the expression frames the usage of the partial F check values.HCRF is able to approximating an advanced distribution the usage of a aggregate of Gaussian density functions.

Christopher Pramerdorfer and Martin Kampel [6] proposed country of the artwork in CNN primarily based totally FER, highlighted key variations among the character works, and in comparison and mentioned their overall performance with a focal point at the underling CNN architectures. They have tested that an ensemble of such CNNs outperforms country of the artwork strategies with out the usage of extra schooling statistics or requiring face registration.

Yimo Guo et al. [7] taken into consideration ranges expression popularity; atlas production level and popularity level. One challenge of the proposed approach is that it's far nevertheless now no longer strong sufficient to conquer demanding situations of sturdy illumination changes. The LDDMM registration set of rules used on this paper won't compensate sturdy illumination changes.

Arushi Raghuvanshi and Vivek Choksi [8] builds mission upon current studies to categorise photographs of human faces into discrete emotion classes the usage of convolutional neural networks (CNNs).

Mercy Rani and Durgadevi [9] proposed a singular face emotion popularity gadget from video frames is proposed. Face place is detected via way of means of Viola Jones set of rules and pores and skin shadeation segmentation with RGB shadeation area is used to extract the face pores and skin and non-pores and skin areas. After the segmentation, morphological operations are implemented to extract the boundary of the non-pores and skin areas particularly eyes and mouth. Then the feelings are identified via way of means of calculating the vicinity of the mouth place.

Saeed Turabzadeh et al. [10] proposed a gadget to assess the FPGA implementation and performance of regression strategies for automated emotional country detection and analysis. The database of 5 customers with 63,000 samples changed into recorded.

\section{III.SOFTWARE REQUIREMENTS}

\section{A. Web Camera:}

A web camera could be a video camera that streams its image in real time to or through a pc network. Web camera is employed to capture the image and 
store in database. Testing image is captured and matched with the database image of an equivalent person.

\section{B. Processor:}

Intel Pentium ahead compatible hardware.

\section{RAM:}

3GB minimum.

\section{Windows 10:}

Windows 10 is a Microsoft product for private computers, and most of the other devices.Microsoft delineate Windows 10 as "operating system as a service" that may receive new updates to its options and functionalities, increased with the potential for enterprise environments to receive non essential updates at a slower speed, or use future support milestones which will solely receive critical updates, reminiscent of security patches, over their five year amount of thought support.

\section{E. Flask:}

Flask is one amongst the micro net framework written in Python.It will be classified as a micro framework as a result, it doesn't need specific tools or libraries. It does not information abstraction layer, type validation or the opposite parts wherever preexisting third-party libraries bestow common functions.

\section{F. Python:}

Python is an interpreter, and high level artificial language with dynamic semantics. Its high-level intrinsical information structures, integrated with dynamic typewriting and dynamic binding, create it alluring for fast application development likewise as to be used as a scripting or glue language to connect existing element together. Python is simple, straightforward to find out syntax streses readability and so cut back the charges of program maintenance. Python supports modules and packages, that motivates

code complexness and code reuse. The python interpreter and python standard library are on the market in resources or binary type without any cost for all major Platforms, and might be freely distributed.

\section{IV.SYSTEM IMPLEMENTATION}

Implementation is the last and most important phase of software development. It means a discussion of building a new system in operation. The new system may be completely new, replacing existing manuals or the default system or it may be a major overhaul of the existing system. Includes user training, system testing and advanced system performance. It is the stage at which text formatting is turned into an active program. Many arrangements are involved before and during the implementation of the proposed plan.Multiscale classifier and inductive learning algorithm Inductive Learning Algorithm (ILA) is a duplicate and instructive machine algorithm used to create a set of distinctive rules, which produces the rules of the form "IF-THEN", with a set of examples, which extract the rules for each iteration and insert a set of rules. The steps in the algorithm are as follows.

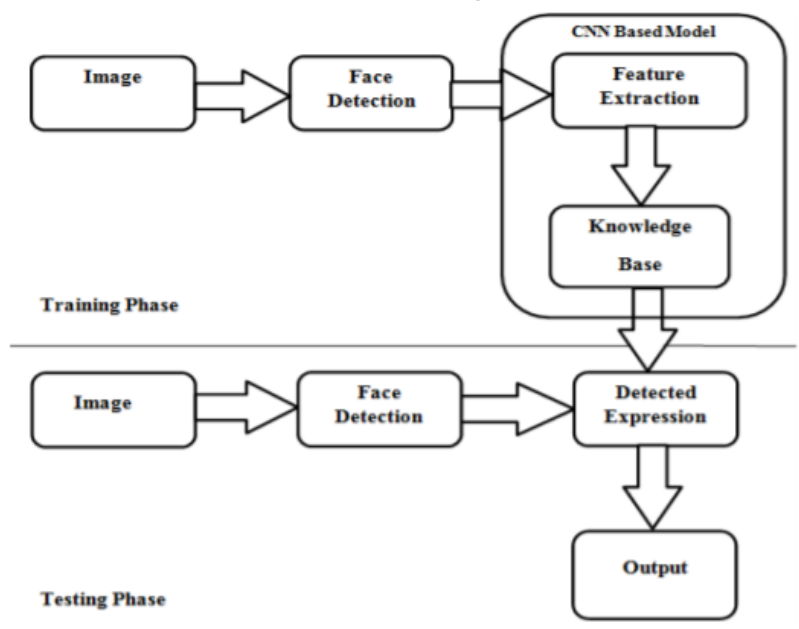

Figure 1 shows the proposed architecture of the system

Step 1: Divide table ' $\mathrm{T}$ ' containing the examples $\mathrm{m}$ into $\mathrm{n}$ tables $(\mathrm{t} 1, \mathrm{t} 2, \mathrm{tn})$. One table for each potential value of the class attribute. (Repeat steps 2-8 for each table) 
Step 2: Generate the value of the attribute combination ' $\mathrm{j}$ ’ $=1$.

Step 3: In a small table on which the work is performed, divide the list of responsibilities into separate sections combination, each combination with different ' $j$ ' symbols.

Step 4: For each combination of symbols, calculate the number of positions of the liability values that appears under the same combination of symbols in the unmarked rows of a small table imaginatively, and at the same time, does not appear under the same combination of the attributes of some small tables. Call the first combination with the maximum number of a large 'MAX' combination occurs.

Step 5: If MAX = = null, increase the ' $j$ ' by 1 and go to Step 3.

Step 6: Mark all the rows of a small table where applicable, where the values of 'MAX' appear, such as separated.

Step 7: Enter the rule (IF attribute = "XYZ" -> THEN the decision says YES / NO) to the R in his left hand the side will have the 'MAX' names with their numbers divided by the AND, and its the right-hand side contains the amount of the decision attribute associated with the sub-table.

Step 8: Once all the lines have been marked as a split, then proceed to process another table below and go to Step 2. alternative, go to Step 4. If no small tables are available, exit the set of rules obtained until then.

\section{A. Data augmentation}

Data augmentation is the process of increasing the amount and diversity of data. There will be no collection of new data, rather transforming the already present data. The most commonly used operations in data augmentation are:

a) Rotation: Rotation operation as the name suggests, just rotates the image by a certain specified degree.

b) Shearing: Shearing is also used to transform the orientation of the image.

c) Zooming: Zooming operation allows us to either zoom in or zoom out. d) Cropping: Cropping allows us to crop the image or select a particular area from an image.

e) Flipping: Flipping allows flipping the orientation of the image. One can use horizontal or vertical flip. One should use this feature carefully as there will be scenarios where this operation might not make much sense e.g. suppose an individual designing a facial recognition system, and then it is highly unlikely that a person will stand upside down in front of a camera, so one can avoid using the vertical flip operation.

f) Changing the brightness level: This feature helps to combat illumination changes. One can encounter a scenario where most of the dataset comprises of images having a similar brightness level e.g. collecting the images of employees entering the office, by augmenting the image make sure that the model is robust and is able to detect the person even in different surroundings.

\section{B. Early Stopping}

The first proposed rules for these problems are based on the analysis of the upper limit of the error made as the function of the iteration number. They provide numerical recurrence number commands that can be calculated before starting the solution process. These early stopping rules work by splitting the original training set into a new training set and a validation set. The error on the validation set is used as a proxy for the generalization error in determining when overfitting has begun. These techniques are often used in training neural networks.

The naive implementation of holdout-based early stopping is summarized as follows:

a) Split the training data into a training set and a validation set, e.g. in a 2-to-1 proportion.

b) Train only on the training set and evaluate the per-example error on the validation set once in a while, e.g. after every fifth epoch.

c) Stop training as soon as the error on the validation set is higher than it was the last time it was checked.

d) Use the weights the network had in that previous step as the result of the training run. 


\section{L2-Regularization}

Regularization is a way of discouraging the weight of the model. It does this by penalizing the loss function. This helps to solve the problem of overfitting. In L2 regularization, it is the square sum of all feature weights as shown in the equation. L2 regularization forces the weights to be small but does not make them zero and does non sparse solution. L2 is not strong to outliers as square terms pushes up the error differences of the outliers and the regularization term tries to correct it by penalizing the weights.

$$
L(x, y) \equiv \sum_{i=1}^{n}\left(y_{i}-h_{\theta}\left(x_{i}\right)\right)^{2}+\lambda \sum_{i=1}^{n} \theta_{i}^{2}
$$

\section{CONCLUSION}

Here we proposed an correct and high-pace facial features popularity machine. The foremost contribution of this machine is that it is able to discover the expression and the effects tested in order that deep CNN's are able to gaining knowledge of facial traits and enhance facial features popularity. Here the Convolutional networks can intrinsically analyze the important facial capabilities via way of means of the use of most effective raw pixel records. We observed how the pixels are being activated in another way relying at the emotion being labelled. The happiness appears to depend upon the pixels related to the eyes and mouth, while the disappointment or the anger appears as an instance to be extra associated with the eyebrows. In this System we've used the XGBoost library. This library implements the gradient boosting selection tree set of rules. Boosting is an ensemble approach in which new fashions are introduced to accurate the mistakes made via way of means of current fashions. By the use of this set of rules we were given one of the quality version named Xception version. Xception is a deep Convolutional neural community structure that includes Depth smart Separable Convolutions. In this version the records first is going thru the access glide, then thru the center glide that is repeated 8 times, and in the end thru the go out glide. Xception version lets in a shorter schooling time on GPUs, extra pictures processing in line with $2 \mathrm{~d}$ in actual-time prediction, and stops over fitting. Because of this one we've were given the coolest accuracy and the expressions are diagnosed accurately.

In future the recognition accuracy may be multiplied via way of means of increasing the information records base via way of means of along with extra face pictures having distinct expressions. Android primarily based totally implementation may be carried out to get the actual time effects. The distinct set of rules also can be used to enhance the accuracy.

\section{REFERENCES}

[1]. Dolan, "The effects of aquarium size and temperature on color vibrancy size and physical activity in bettasplendens", 2015.

[2]. S. Sharpe, Aquarium Nitrogen Cycle: How an Aquarium Cycles, 2018, onlineAvailable: https://www.thesprucepets.com/aquariumnitrogen-cycle-1378370.

[3]. C. Dupont, P. Cousin and S. Dupont, "IoT for aquaculture 4.0 smart and easy-to-deploy realtime water monitoring with IoT", Proc. Global Internet Things Summit (GIoTS), pp. 1-5, Jun. 2018.

[4]. T. I. Salim, T. Haiyunnisa and H. S. Alam, "Design and implementation of water quality monitoring for eel fish aquaculture an examination of microbubble aeration", Proc. Int. Symp. Electron. Smart Devices (ISESD), pp. 2930, Nov. 2016.

[5]. K. M. Stehfest, C. G. Carter, J. D. McAllister, J. D. Ross and J. M. Semmens, "Response of atlantic salmon salmo salar to temperature and dissolved 
oxygen extremes established using animal-borne environmental sensors", Nature, vol. 7, pp. 4545, 2017.

[6]. J.-H. Chen, W.-T. Sung and G.-Y. Lin, "Automated monitoring system for the fish farm aquaculture environment", Proc. IEEE Int. Conf. Syst. Man Cybern., pp. 1161-1166, Oct. 2015.

[7]. C. Encinas, E. Ruiz, J. Cortez and A. Espinoza, "Design and implementation of a distributed IoT system for the monitoring of water quality in aquaculture", Proc. Wireless Telecommun. Symp. (WTS), pp. 1-7, Apr. 2017.

[8]. Y.-B. Lin, Y.-W. Lin, C.-M. Huang, C.-Y. Chih and P. Lin, "IoTtalk: A management platform for reconfigurable sensor devices", IEEE Internet Things J., vol. 4, no. 5, pp. 1152-1562, Oct. 2017.

[9]. G. Farmer, CO2: Striking the Balance, 2016, onlineAvailable:

https://www.practicalfishkeeping.co.uk/features/ articles/co2-striking-the-balance.

[10].J. E. Bly and W. L. Clem, "Temperature and teleost immune functions", Fish Shellfish Immunol., vol. 2, no. 3, pp. 159-171, 1992.

[11].T. Masaharu et al., "Effect of temperature on survival growth and malformation of cultured larvae and juveniles of the seven-band grouper epinephelus septemfasciatus", Fisheries Sci., vol. 80, no. 1, pp. 69-81, 2014.

[12].The Importance of Oxygen in the Aquarium, 2018, onlineAvailable: https://www.algone.com/oxygen-in-theaquarium.

[13].Your Guide to Ammonia Toxicity, 2011, onlineAvailable:

http://www.aquariumadvice.com/forums/f12/yo ur-guide-to-ammonia-toxicity-159994.html.

[14].T. Sarac, Electrical Conductivity in Freshwater Aquariums, 2018, onlineAvailable: http://fluvalaquatics.com/ca/explore/did-youknow/equipment/155-electrical-conductivitymonitoring-system-part-two/\#.XAL_hzgzZ0x.
[15].What You Need to Know About Reverse Osmosis, 2016,onlineAvailable: https://www.practicalfishkeeping.co.uk/features/ articles/what-you-need-to-know-about-reverseosmosis.

\section{Cite this article as :}

Ashna Namira, Aravind Naik, Nikhil Floyd Dsouza, "Face Expression Recognition using CNN", International Journal of Scientific Research in Computer Science, Engineering and Information Technology (IJSRCSEIT), ISSN : 2456-3307, Volume 7 Issue 4, pp. 235-240, July-August 2021. Available at doi : https://doi.org/10.32628/CSEIT217451 Journal URL : https://ijsrcseit.com/CSEIT217451 